\title{
¿SE JUSTIFICA EL TRATAMIENTO DE LA RECIDIVA LOCAL DEL CÁNCER DE RECTO?*
}

\author{
Dr. Guillermo Bannura C.
}

1 Servicio y Departamento de Cirugía. Hospital Clínico San Borja Arriarán. Campus Centro, Facultad de Medicina, Universidad de Chile. Santiago, Chile.

\begin{abstract}
\section{Treatment of local relapses of rectal cancer}

The treatment of local relapses of rectal cancer requires a multimodal strategy. The preoperative assessment should consider the performance status of the patient, determine the precise anatomical involvement and look for distant metastases. The risk factors for a local relapse are the height of the tumor, the type of intervention, an elevated carcinoembryonic antigen in the preoperative period, the circumferential margin involvement and the stage of the tumor. The prognostic factors for an established local relapse are the type of surgery for the primary tumor (local excision, anterior or abdominoperineal resection), the type of relapse (staging), the presence of symptoms (profound pelvic pain) and specially the possibility of achieving a complete resection. A systematic analysis of 55 reports concluded that a radical resection with curative intention was achieved in $40 \%$ of cases. Preoperative external or intraoperative radiotherapy was used in 12 to $97 \%$ and 8 to $91 \%$ of cases respectively. Thirty days postoperative mortality was $2.2 \%$, the rates of local control of the tumor ranged from 29 to $100 \%$ and five years survival was at least $25 \%$ with a superior limit of $41 \%$. Although there are no clear guidelines for the management of these patients, there is a growing consensus in the use of preoperative radiotherapy to achieve better surgical results. Therefore a multimodal treatment of local relapses is justified.
\end{abstract}

Key words: Rectal cancer, local relapse, surgery.

\section{Resumen}

El tratamiento actual de la recidiva local (RL) del cáncer de recto representa un verdadero desafío para el equipo multidisciplinario que debe manejar esta seria complicación con una estrategia de terapia multimodal. La evaluación preoperatoria de la RL considera el status perfomance del paciente y busca establecer el compromiso anatómico preciso, así como descartar enfermedad a distancia. Entre los factores de riesgo para la ocurrencia de una RL destacan la altura del tumor (bajo), el tipo de intervención (RAP), el CEA preoperatorio elevado, la perforación intraoperatoria, el compromiso del margen circunferencial y el estadio del tumor

*Recibido el 21 de abril de 2014 y aceptado para publicación el 12 de junio de 2014.

El autor no refiere conflictos de interés.

Correspondencia: Dr. Guillermo Bannura C.

gbannura@gtdmail.com 
primario. Entre los factores pronósticos de una RL ya establecida se incluye el tipo de cirugía del primario (resección local, resección anterior, RAP), el tipo de RL (estadificación), la presencia de síntomas (el dolor pélvico profundo) y especialmente la posibilidad de lograr una resección R0 de la RL. Una revisión sistemática reciente sobre 55 estudios muy heterogéneos concluye que la resección radical con intención curativa se logró en el $40 \%$ de los casos; la radioterapia externa preoperatoria al abordaje de la RL en dosis de 40 a 60 Gy se empleó en el 12 al 97\% de los casos y la IORT varió entre el 8 y el 91\% con dosis entre 10 y 20 Gy; la mortalidad operatoria a 30 días fue de $2,2 \%$ como promedio (extremos $0-8,8 \%$ ); la tasa de control local en los pacientes R0 varió entre 29 y $100 \%$ y la sobrevida a 5 años fue al menos $25 \%$ con un límite superior de $41 \%$. No existe una evidencia categórica sobre cual es la estrategia terapéutica más adecuada en el manejo de una RL. Sin embargo, existe un consenso creciente que estos pacientes deben recibir un esquema de RQT preoperatoria con el fin de disminuir el tamaño y/o el estadio de la recidiva que permita alcanzar una resección R0. Los resultados actuales sugieren que el tratamiento multimodal se justifica razonablemente en el manejo de la RL, que la selección de pacientes es crucial y que se requiere un equipo altamente capacitado en cirugía pélvica radical de salvataje.

Palabras clave: Recurrencia local, cáncer de recto, tratamiento multimodal.

\section{Introducción}

La recidiva local o loco-regional (RL) de un cáncer de recto corresponde a la aparición de enfermedad neoplásica en la pelvis luego de la extirpación del tumor primario con intención curativa. La incidencia reportada en las décadas pasadas de esta complicación del tratamiento quirúrgico del adenocarcinoma rectal fluctuaba entre 5 y $50 \%{ }^{1}$, variabilidad que se explica por la heterogeneidad de las series y la calidad de la cirugía misma. Desde la introducción en el mundo Occidental de la resección total del mesorrecto (TME) y el uso habitual de la radioquimioterapia (RQT) preoperatoria, las cifras de RL han bajado considerablemente a cifras que varían entre 6 y $12 \%{ }^{2}$. Empleando una estrategia diferente, autores japoneses publican resultados similares en RL y sobrevida mediante cirugía radical que incluye la linfadenectomía pélvica lateral sin el empleo de terapia radiante ${ }^{3}$. La sobrevida media de los pacientes con RL sin tratamiento es de 6 a 7 meses con morbilidad muy severa. La radioterapia con o sin quimioterapia aumenta la supervivencia a 10 a 17 meses con alivio sintomático en un tercio de los pacientes ${ }^{4}$.

El tratamiento actual de la RL representa un verdadero desafío para el equipo multidisciplinario que debe manejar esta seria complicación con una estrategia de terapia multimodal, que incluye la RQT neoadyuvante, la cirugía radical (salvataje), la radioterapia intraoperatoria y la quimioterapia adyuvante. El progreso y refinamiento de los procedimientos quirúrgicos, la experiencia acumulada y el apoyo de las unidades de cuidados intensivos ha permitido un control adecuado de la mortalidad operatoria, por lo que actualmente se acepta que la resección radical de la enfermedad pelviana es la única alternativa de curación que tiene un paciente con una RL, aunque el precio es una elevada morbilidad perioperatoria. Aún en pacientes cuidadosamente seleccionados en centros calificados, la cifra de resección completa con intención curativa no alcanza el 50\% de los casos sometidos a exploración quirúrgica por una $\mathrm{RL}^{5}$. El pronóstico de los pacientes en los cuales no es posible la resección es ominoso y la calidad de vida es habitualmente deplorable, con períodos de supervivencia que en ocasiones supera los 8 a 12 meses.

\section{Evaluación preoperatoria de la RL}

Aparte de considerar las condiciones generales y nutritivas, las condiciones co-mórbidas y la reserva fisiológica del paciente, lo esencial es establecer un diagnóstico histológico y/o por imágenes de la RL y el compromiso anatómico lo más detallado posible, así como descartar enfermedad a distancia. Esto incluye el estudio metabólico habitual con parámetros nutricionales (albúmina, recuento de linfocitos), el antígeno carcinoembrionario (CEA), la rectoscopía o la colonoscopía con biopsia y los estudios por imágenes. La tomografía computada (TC) tiene una sensibilidad que no supera el $70 \%$ y una especificidad del $85 \%$, dada la dificultad para discriminar entre tumor y fibrosis y permite mostrar eventualmente focos a distancia (hígado, pulmón), por lo que se recomienda una TC de tórax, abdomen y pelvis ${ }^{6}$. La resonancia magnética $(\mathrm{RM})$ de la pelvis es más sensible para precisar el compromiso de los órganos pelvianos pero tiene limitaciones en los pacientes irradiados ${ }^{7}$. El PET-TC indica la actividad metabólica de una lesión sospechosa y en la pelvis tiene un rendimiento del $87 \%$, aún en pacientes irradiados, pero falla en la detección de metástasis pequeña y/o de baja actividad metabólica ${ }^{8}$ (Figura 1). 


\section{Factores predictivos de la ocurrencia de una RL}

\section{1) Factores de la cirugía primaria}

a. La localización del tumor primario por debajo de la reflexión peritoneal es considerado por algunos autores como un factor predictivo independiente de RL, aunque esto es controversial ${ }^{9}$.

b. Tipo de cirugía. Los pacientes sometidos a una resección abdominoperineal (RAP) tienen una tasa de RL mayor que la resección anterior baja, lo que probablemente refleja un sesgo de selección ${ }^{10}$. El riesgo de RL de la resección interesfintérica en tumores muy bajos es en la actualidad motivo de discusión ${ }^{11}$. En el extremo del abanico, los pacientes con una RL luego de resección local, tienen un mejor pronóstico relativo y mayor posibilidad de lograr una resección R0.

c. Tipo de resección. Basado en el estudio de la pieza operatoria se clasifica en R0 (no hay tumor residual) que se considera curativa ${ }^{12}$, mientras que R1 (enfermedad residual microscópica) y R2 (enfermedad residual macroscópica) son paliativas, situación que se puede compensar en parte con la RQT adyuvante.

d. La perforación intraoperatoria del tumor se considera un factor de riesgo tanto de RL (la tasa de RL se eleva de 10 a $29 \%)^{13}$ como de recidiva a distancia con una reducción de la supervivencia ${ }^{14}$, lo que podría mejorar con la neoadyuvancia.

\section{2) Factores patológicos}

a. Incluyen el estadio tumoral, el factor $\mathrm{T}$ (penetración en la pared), la configuración del borde del tumor y los brotes tumorales (budding). Los más significativos son el compromiso del margen radial o circunferencial (CRM) descrito por Quirke ${ }^{15}$, la invasión linfovascular, el factor T, la invasión venosa extramural y un pobre grado de diferenciación tumoral ${ }^{16}$. Un CRM positivo se define como la extensión tumoral y linfonodos positivos dentro de $1 \mathrm{~mm}$ del CRM, que muestra una RL de $37 \%$ vs $8 \%$ con un CRM negativo ${ }^{17}$. La QRT preoperatoria podría reducir el riesgo de CRM positivo y la RQT adyuvante potencialmente reduce la RL luego de una CRM positivo.

b. Margen de resección distal: desde la regla de los $5 \mathrm{~cm}$ se ha cambiado a un margen de $1 \mathrm{~cm}$ en la pieza fresca, lo que también depende en parte de las terapias neoadyuvantes, lo que ha permitido una cirugía con conservación de esfínteres en muchos $\operatorname{casos}^{18}$.

c. CEA. La reducción de un CEA preoperatorio elevado es un buen indicador de cirugía completa y augura un buen pronóstico a largo plazo, mientras que los pacientes que mantienen niveles elevados posquirúrgicos están más propensos a presentar metástasis y $\mathrm{RL}^{19}$.

\section{3) Factores terapéuticos}

a. Tanto la QRT neoadyuvante como la adyuvante reducen la tasa de RL en forma significativa, motivo por el cual el esquema preoperatorio se ha transformado en el estándar actual del manejo del cáncer de recto bajo localmente avanzado (T3$\mathrm{T} 4$, y/o cualquier $\mathrm{T}$ con $\mathrm{LN}+$, es decir, estadios II y III $)^{2}$.

\section{Factores pronósticos de la RL establecida}

\section{Tipo de cirugía inicial}

Los pacientes inicialmente sometidos a una resección anterior tienen una mejor resecabilidad y sobrevida que los pacientes con una RAP. En el primer caso, el diagnóstico suele ser más precoz mediante el tacto rectal y/o la biopsia endoscópica a nivel de la anastomosis. En los casos de RAP, el dolor pelviano usualmente sugiere la presencia de una masa central en la pelvis de difícil manejo ${ }^{20}$.

\section{Tipo de RL (estadificación)}

Existen varias clasificaciones de la RL que orientan hacia la posibilidad de lograr una resección R0. En la Clínica Mayo la RL se gradúa según el número de puntos fijos y los síntomas, siendo peor el pronóstico en los pacientes con dolor pélvico y con más de un punto de fijación ${ }^{21}$. El Memorial Sloan Kettering define la RL como local (luego de resección local endoanal), axial anastomótica (luego de una resección anterior), posterior con infiltración de sacro-coxis, anterior (con invasión de órganos de la esfera urogenital) y lateral (compromiso de la pared lateral de la pelvis) ${ }^{22}$. Yamada ${ }^{23}$ las divide en RL localizada (adyacente a órganos pelvianos o tejido conectivo de soporte), sacral (invasión de S3 a S5 y/o coxis) y lateral (compromiso del nervio ciático, sacro alto y pared pélvica lateral). En Leeds ${ }^{24}$ distinguen la RL central (confinada los órganos pélvicos), lateral (estructuras linfovasculares y ligamentosas), presacra (compromiso del hueso sacro) y compuesta (sacro y paredes laterales). En general, en casos de RL luego de una resección local es factible lograr una resección R0. En los casos de RL luego de una resección anterior puede rescatarse mediante una RAP o una exenteración posterior o total, mientras que la RL luego de una RAP es de difícil rescate por el compromiso de la pared lateral de la pelvis. Así, Zhao y cols ${ }^{12}$, logran una resección R0 en el $85 \%$ de la RL axial versus $33 \%$, $25 \%$ y $4,3 \%$ en las RL anteriores, posteriores y laterales, respectivamente. 


\section{Síntomas acompañantes de la $R L$}

El dolor pélvico es el síntoma más común y destacado asociado a una RL, a veces con irradiación a las extremidades inferiores ${ }^{4,5,12}$. Generalmente se correlaciona con un tumor extenso y de peor pronóstico que una RL asintomática (aproximadamente un tercio de los pacientes son asintomáticos al momento del diagnóstico).

\section{Factores quirúrgicos}

Lograr una resección $\mathrm{R} 0$ es el factor pronóstico más importante, independiente incluso del tipo de RL y se logra en alrededor del $50 \%$ de los pacientes intervenidos, es decir, alrededor del $20 \%$ del global de pacientes con diagnóstico de una $\mathrm{RL}^{4,5,12,17,20-26}$. Se considera una contraindicación formal de cirugía de salvataje los pacientes con pelvis congelada con compromiso de la pared lateral, de la escotadura ciática, de los vasos ilíacos con edema de la extremidad, la invasión de S1-S2 y la presencia de enfermedad diseminada ${ }^{27}$.

\section{La RQT neoadyuvante y la RQT adyuvante}

Como terapia exclusiva han mostrado resultados pobres en el control de la enfermedad con sobrevida a 5 años cercana a $0 \%$. La RQT neoadyuvante se considera como parte del tratamiento actual en los pacientes que van a ser sometidos a una laparotomía exploradora con intención de resección radical e intención curativa ${ }^{4,5,12,27,28}$. Desgraciadamente, una proporción no menor de estos pacientes han sido sometidos a RQT preoperatoria del tumor primario, lo que limita la posibilidad de utilizarla en el manejo de la RL.

\section{La radioterapia intraoperatoria (IORT)}

Es una forma de braquiterapia que aumenta en 3 veces el efecto biológico de la radioterapia externa, reduciendo las complicaciones asociadas a la radioterapia. Se ajusta la dosis de 15 a 30 Gy a un área específica y podría mejorar los resultados de la cirugía resectiva lo que es controversial ${ }^{29}$.

\section{Resultados del tratamiento multimodal con intención curativa de la RL}

Una revisión sistemática reciente que incluye 55 estudios sobre 3.767 pacientes, analiza los resultados del tratamiento de la RL $(481 / 1.188=40 \%)$ con intención curativa. Las series son heterogéneas, los protocolos de estudio, las terapias neo o adyuvantes así como el tipo de RL y la cirugía efectuada son extremadamente variados $\mathrm{y}$, por último, el seguimiento es muy variable ${ }^{29}$. Considerando esto, podemos resumir que la principal cirugía efectuada en la operación índice fue la RAP en el 27\% (extremos
$0-100 \%$ ); la radioterapia externa preoperatoria fue entregada en el $28 \%$ de los casos (extremos $0-100 \%$ ) y la quimioterapia en el 37\% (extremos $0-100 \%$ ). En cuanto a la presentación clínica al momento del diagnóstico el $29 \%$ de los casos era asintomático y $50 \%$ tenían síntomas con la presencia de dolor pélvico. El intervalo entre la cirugía primaria y el diagnóstico de la RL fluctuó entre 12 y 44 meses. Hubo recidiva a distancia concomitante en el 2 a $41 \%$ de los pacientes, de las cuales sólo 3 a 16\% fueron resecadas con intención curativa. En el 17 a 99\% de los casos se intentó una cirugía resectiva con intención curativa, diferencia que se debe a la exclusión en algunas series de los pacientes con enfermedad irresecable demostrada ya sea en el estudio preoperatorio o en la laparotomía exploradora. El porcentaje de pacientes resecados fue $81,9 \%(3.088 / 3.767)$ y el promedio de resecciones R0 fue 59\% (extremos $14-100 \%$ ). El sacro fue resecado en el $14 \%$ de 20 estudios (extremos 2-73\%). La radioterapia externa preoperatoria al abordaje de la RL en dosis de 40 a 60 Gy fue usada entre el 12 y $97 \%$ de los casos y la IORT varió entre el 8 y el $91 \%$ con dosis entre 10 y $20 \mathrm{~Gy}$. La mortalidad operatoria a 30 días fue de $2,2 \%$ como promedio (extremos $0-8,8 \%$ ). La tasa de control local en los pacientes R0 varió entre 29 y $100 \%$ y la sobrevida a 5 años fue al menos $25 \%$ con un límite superior de $41 \%$.

En suma, esta revisión sistemática, con las limitaciones señaladas, sugiere que al menos el $40 \%$ de los pacientes no seleccionados portadores de una RL pueden ser candidatos a un tratamiento con intención curativa. Sólo un pequeño porcentaje de pacientes con metástasis a distancia sincrónica con la RL obtienen este beneficio. El dolor intratable se asocia con una recidiva en la pelvis probablemente incurable. Los predictores más destacados de lograr una resección R0 por una RL son la resección anterior (contrario al paciente sometido a una RAP) y la ausencia de dolor pélvico al momento del diagnóstico de la RL. No existe una evidencia categórica sobre cual es la estrategia terapéutica más adecuada en el manejo de una RL. Sin embargo, existe consenso creciente que estos pacientes deben recibir un esquema de RQT preoperatoria con los fines de disminuir el tamaño y/o el estadio de la recidiva que permita alcanzar una resección $\mathrm{R} 0^{28}$. La mortalidad operatoria está controlada en centros con experiencia, aunque existe un costo en la morbilidad postoperatoria que no es menor. En la Tabla 1 se muestran los resultados de algunas publicaciones recientes. Esta mejoría discreta de los resultados globales con una sobrevida promedio del $30 \%$ de los pacientes resecados se debe a una mejor selección de los pacientes y al impacto del manejo multimodal. En los pacientes con una metástasis a distancia sin- 
Tabla 1. Resultados de la cirugía de salvataje en el manejo de la RL

\begin{tabular}{|c|c|c|c|c|c|}
\hline Autor, año & $\mathbf{n}$ & Ro \% & $\begin{array}{c}\text { Mortalidad } \\
\text { operatoria }\end{array}$ & $\begin{array}{c}\text { Sobrevida } 5 \text { años } \\
\text { R0 \% }\end{array}$ & $\begin{array}{c}\text { Follow up } \\
\text { promedio meses }\end{array}$ \\
\hline García-Aguilar, 2001 30 & 51 & 82 & 0 & 35 & 28 \\
\hline Moriya, $2004^{31}$ (abdomino-sacra) & 57 & 84 & 3,5 & 42 & 42 \\
\hline Bedrosian , 2006 25 & 134 & 48,5 & ns & 43 & 43 \\
\hline Heriot, $2008^{32}$ & $152 *$ & 61 & 0,6 & 46 & 20 \\
\hline Wiig, $2008^{33}$ & 124 & 42 & 0,7 & 52 & 23 \\
\hline Kusters, $2009^{34}$ & 70 & 54 & 8,2 & 40,5 & 35 \\
\hline Hansen, $2009^{35}$ & 185 & 52 & 1,5 & 55 & 44 \\
\hline Pacelli, $2010^{36}$ & 44 & 64 & 6,8 & 72,4 & 82 \\
\hline Jiang, $2011^{37}$ & 187 & 47 & 2,7 & 42,6 & 60 \\
\hline
\end{tabular}

crónica parece mejor utilizar un esquema de quimioterapia de inducción previo al tratamiento radiante, aunque no hay evidencia definitiva. La posibilidad de rescate de un paciente con una RL sometido a neoadyuvancia con RQT y cirugía radical del tumor primario probablemente se reduce si se compara con un paciente tratado con cirugía exclusiva. La magnitud del procedimiento puede ir desde la simple laparotomía exploradora hasta la exenteración pélvica total, lo que requiere de un equipo quirúrgico altamente capacitado en este tipo radical de cirugía de salvataje (Figura 2).

\section{Experiencia en el HCSBA}

En un período de 25 años se ha tratado con cirugía radical 382 pacientes portadores de un cáncer de recto con intención curativa (se excluyen los pacientes sometidos a una resección local). La RL global es $12 \%$, es decir, 45 pacientes, de los cuales 21 fue sometido a una cirugía por una RL. Se trata de 9 hombres y 12 mujeres, con un promedio de edad de 58 años (extremos 35-75). La intervención primaria fue la resección anterior en 12 casos (1 cistectomía parcial), la RAP en 4 y la exenteración pélvica posterior en 5 ( 1 cistectomía parcial). De ellos, 4 pacientes habían recibido RQT neoadyuvante y 3 recibieron RQT postoperatoria. La presentación clínica fue la estenosis con tumor rectal en 7 casos, masa perineal en 5, obstrucción intestinal en 2 , hemorragia digestiva baja masiva en 1 , dolor pélvico intratable en 4 , fístula entero-colónica en 1 (Figura 1) e hidroureteronefrosis en 1. El intervalo entre la cirugía primaria y el diagnóstico de la RL

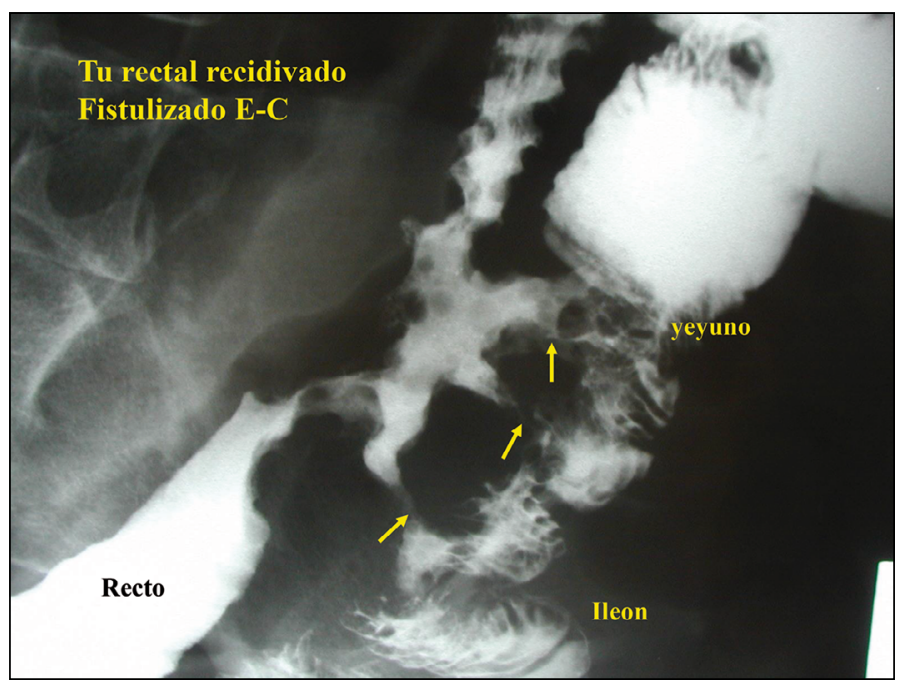

Figura 1. Enema Baritado DC: Tu rectal recidivado y fistulizado: doble fístula al yeyuno $\mathrm{y}$ al íleon. 


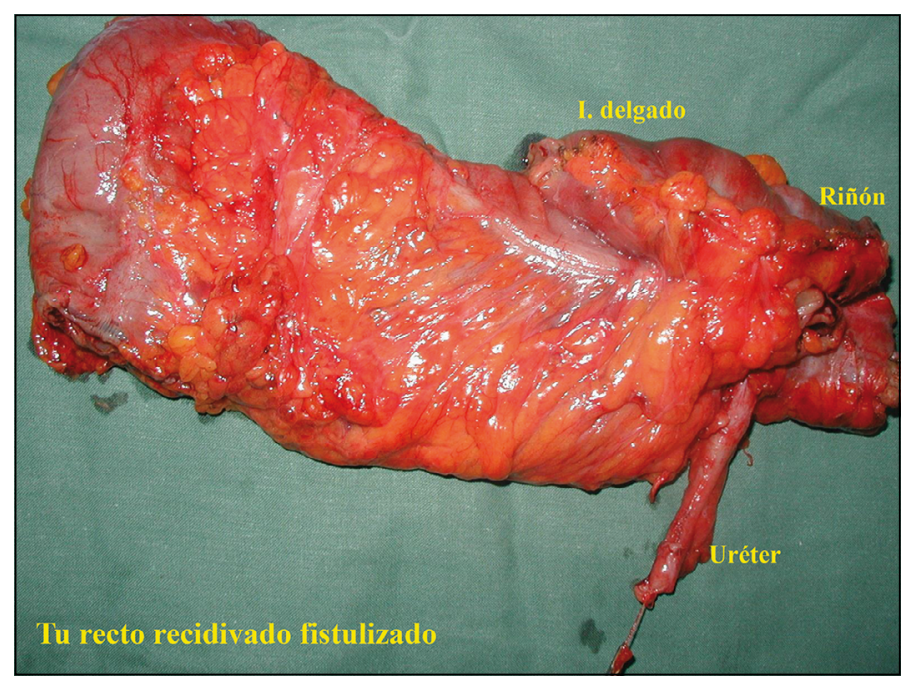

Figura 2. Pieza operatoria: incluye recto, colon, intestino delgado y riñón con uréter izquierdos.

Tabla 2. Cirugía y resultados oncológicos en 21 pacientes tratados por una RL

\begin{tabular}{|lcccc|}
\hline Intervención & Paliativa & Curativa & $\begin{array}{c}\text { Vivos } \\
\text { (meses) }\end{array}$ & $\begin{array}{c}\text { Fallecidos } \\
\text { (meses) }\end{array}$ \\
Resección perineal + vagina & & 2 & 120 & $66^{*}$ \\
Resección abdomino-perineal & 4 & 1 & 92 & $24 ; 28 ; 15 ; 13$ \\
Resección anterior ampliada (riñón: 2; vejiga: 1; intestino delgado: 2) & & 6 & $140 ; 120 ; 116$ & $60 ; 21 ; 19$ \\
Exenteración pélvica total & 1 & & $24^{\&}$ \\
Resección recidiva + intestino delgado (1) + vejiga (1)+ hígado (2) & 4 & & $18 ; 12 ; 5 ; 16$ \\
Laparotomía exploradora & 3 & & $6 ; 17 ; 11$ \\
Total & 12 & 9 & $5(40 \%)$ & \\
\hline
\end{tabular}

*Metástasis hepática. ${ }^{\&}$ Carcinomatosis peritoneal.

fue 32 meses como promedio (extremos 10-108). La evaluación preoperatoria incluyó la TC en 17 pacientes, la endoscopía con biopsia en 10, biopsia quirúrgica en 2 y la ecografía en 1 . El estadio patológico del tumor primario fue IIA en 7 casos, IIB en 1, IIIA en 7 y IIIC en 6. La cirugía efectuada y los resultados oncológicos se detallan en la Tabla 2. La morbilidad postoperatoria afectó al $40 \%$ de los pacientes (13 complicaciones en 10 pacientes), incluyendo 3 casos de ISO de la herida perineal, 1 lesión de uréter (requirió una nefrectomía), 1 dehiscencia anastomótica que se resolvió con una ileostomía en asa, 1 hemoperitoneo (reoperación precoz), 1 trombosis venosa profunda, 2 casos de neumopatía y 4 pacientes con una vejiga neurogénica e infección urinaria asociada. En suma, hubo 3 reoperados, la estadía hospitalaria fue en promedio de 24,8 días (extremos 8-64) y no hubo mortalidad operatoria.
Seis pacientes recibieron RQT preoperatoria y 1 postoperatoria. En cuanto a sobrevida, hay 5 vivos sin evidencias de enfermedad de los 9 pacientes sometidos a una cirugía con intención curativa (40\%) con un seguimiento promedio de 84 meses. Los pacientes sometidos a una resección paliativa tuvieron una sobrevida promedio de 16 meses (extremos 5-24) versus 11 meses (extremos 6-17) en los casos de laparotomía exploradora exclusiva.

\section{Referencias}

1. Phillips RK, Hittinger R, Blesovsky L, Fry JS, Fielding LP. Local recurrence following 'curative' surgery for large bowel cancer: II. The rectum and rectosigmoid. Br J Surg. 1984;71:17-20.

2. Sauer R, Liersch T, Merkel S, Fietkau R, Hohenberger 
W, Hess C, et al. Preoperative versus postoperative chemoradiation for locally advanced rectal cancer: results of the German CAO/ARO/AIO-94 randomized phase III trial after a median follow up of 11 years. Clin Oncol. 2012;30:1926-33.

3. Konishi T, Watanabe T, Nagawa H, Ueno M, Kuroyanagi $\mathrm{H}$, Fujimoto $\mathrm{Y}$, et al. Preoperative chemoradiation and extended pelvic lymphadenectomy for rectal cancer. Two distinct principles. World Gastrointest Surg. 2010;2:95-100.

4. Palmer G, Martling A, Cedermark B, Holm T. A population-based study on the management and outcome in patients with locally recurrent rectal cancer. Ann Surg Oncol. 2007;14:447-54.

5. Enríquez-Navascués JM, Borda N, Lizerazu A, Placer C, Elosegui JL, Ciria JP, et al. Patterns of local recurrence in rectal cancer after a multidisciplinary approach. World J Gastroenterol. 2011;17:1674-84.

6. Beets-Tan RG, Beets GL, Borstlap AC, Oei TK, Teune TM, Von Meyenfeldt MT, et al. Preoperative assessment of local tumor extent in advanced rectal cancer. CT or high-resolution MRI? Abdom Imaging. 2000;25:533-41.

7. Messiou C, Chalmers A, Boyle K, Wilson D, Sagar P. Preoperative MR assessment of recurrent rectal cancer. Br J Radiol. 2008;966:468-73.

8. Schaefer O, Langer M. Detection of recurrent rectal cancer with CT, MRI and PET/CT. Eur Radiol. 2997; 17:2044-54.

9. Faerden AE, Naimy N, Wiik P. Total mesorectal excision for rectal cancer: difference in outcome for low and high rectal cancer. Dis Colon Rectum 2005;48:2224-31.

10. Yang Z, Xu H, Zhang W, Xu Y, Xu Z. A retrospective analysis of ultralow anterior resection vs abdominoperineal resection for low rectal cancer. Hepatogastroenterology 2012;59:1780-3.

11. Martin ST, Heneghan HM, Winter DC. Systematic review of outcomes after intersphincteric resection for low rectal cancer. Br J Surg. 2012;99:603-12.

12. Zhao J, Du C-Z, Sun Y-S, Gu J. Patterns and prognosis of locally recurrent rectal cancer following multidisciplinary treatment. World J Surg. 2012;18:7015-20.

13. Eriksen MT, Wibe A, Syse A, Haffner J, Wiig JN. Norwegian rectal Cancer Group; Norwegian Gastrointestinal Cancer Group: Inadvertent perforation during rectal cancer resection in Norway. Br J Surg. 2004;91:210-6.

14. Bülow S, Christensen IJ, Iversen LH, Harling H; Danish Colorectal cancer Group. Intraoperative perforation is an important predictor of local recurrence and impaired survival after abdominoperineal resection for rectal cancer. Colorectal Dis. 2011;13:1256-64.

15. Quirke P, Durdey P, Dixon MF, Williams NS. Local recurrence of rectal adenocarcinoma due to inadequate surgical resection. Histopathological study of lateral tumour spread and surgical excision. Lancet 2986;2:9969.
16. Parfitt JR, Driman DK. The total mesorectal excision specimen for rectal cancer: a review of its pathological assessment. J Clin Pathol. 2009;60:849-55.

17. Gosens MJ, Klaassen RA, Tan-Go I, Rutten HJ, Martijn H, Van der Brule AJ, et al. Circumferential margin involvement is the crucial prognostic factor after multimodality treatment in patients with locally advanced rectal carcinoma. Clin Cancer Res. 2007;13:6617-23.

18. Rutkowski A, Nowacki MP, Chwalinski M Oledzki J, Bednarczyk M, Liszka-Dalecki P, et al. Acceptance of a 5-mm distal bowel resection margin for rectal cancer: is it safe? Colorectal Dis. 2012;14:71-8.

19. Lin JK, Lin CC, Yang SH, Wang HS, Jiang JK, Lan YT, et al. Early postoperative CEA level is a better prognostic indicator than is preoperative CEA level in predicting prognosis of patients with curable colorectal cancer. Int J Colorectal Dis. 2011;54:901-11.

20. Wanebo HJ, Antouik P, Koness RJ, Levy A, Vezeridis M, Cohen SI, Wrobleski DE. Pelvic resection of recurrent rectal cancer: technical considerations and outcomes. Dis Colon Rectum 1999;42:1438-48.

21. Hahnloser D, Nelson H, Gunderson LL, Hassan I, Haddock MG, O'Connelly MJ. Curative potential of multimodality therapy for locally recurrent rectal cancer. Ann Surg. 2003;237:502-8.

22. Moore HG, Shoup M, Riedel E, Minsky BD, Alektiar KM, Ercolani M, et al. Colorectal cancer pelvic recurrences: determinants of resecability. Dis Colon Rectum 2004;47:1599-606.

23. Yamada K, Ishizawa T, Niwa K Patterns of pelvic invasion are prognostic in the treatment of locally recurrent rectal cancer. Br J Surg. 2001;88:988-93.

24. Mirnezami AH, Sagar PM, Kavanagh D, Witherspoon D, Lee P, Winter P. Clinical algorithms for the surgical management of locally recurrent rectal cancer. Dis Colon Rectum 2010;53:1248-57.

25. Bedrosian I, Giacco G, Pederson L, Rodríguez-Bigas MA, Feig B, Hunt KK, et al. Outcome after curative resection for locally recurrent rectal cancer. Dis Colon Rectum 2006;49:175-81.

26. Lizarazu A, Enríquez-Vanascués JM, Placer C, Carrillo A, Sainz-Lete A, Elósegui JL. Abordaje quirúrgico de la recidiva locorregional del cáncer de recto. Cir Esp. 2011;89:269-74.

27. Cai Y, Li Z, Gu X, Fang Y, Xiang J, Chen Z. Prognostic factors associated with locally recurrent cancer following primary surgery (review). Oncology letters 2014;7:10-6.

28. Tanis PJ, Doeksen A, Van Lanschot JJB. Intentionally curative treatment of locally recurrent rectal cancer: a systematic review. Can J Surg. 2013;56:135-44.

29. Dresen RC, Gosens MJ, Martjin H, Nieuwenhuijzen GA, Creemers GJ, Daniels-Gooszen AW, et al. Radical resection after IORT-containing multimodality treatment is the most important determinant for outcome in patients treated for locally recurrent rectal cancer. Ann 
Surg Oncol. 2008;15:1937-47.

30. García-Aguilar J, Cromwell JW, Marra C, Lee SH, Madoff RD, Rothenberger DA. Treatment for locally recurrent rectal cancer. Dis Colon Rectum 2001;44:1743-8.

31. Moriya Y, Akasu T, Fujita S, Yamamoto S. Total pelvic exenteration with distal sacrectomy for fixed recurrent rectal cancer in the pelvis. Dis Colon Rectum 2004;47:2047-53.

32. Heriot AG, Tekkis PP, Darzi A, Mackay J. Surgery for local recurrence of rectal cancer. Colorectal Dis. 2006;8:733-47.

33. Wiig JN, Larsen SG, Dueland S, Giercksky KE. Prognostic factors after preoperative irradiation and surgery for locally advanced rectal cancer. Eur J Surg Oncol. 2008;34:410-7.

34. Kusters M, Dresen RC, Martijn H, Nieuwenhuijzen GA, van de Velde CJ, van den Berg HA, et al. Radicality of resection and survival after multimodality treatment is influenced by subsite of locally recurrent rectal cancer. Int J Radiat Oncol Biol Phys. 2009;75:1444-9.

35. Hansen MH, Balteskard L, Dorum LM, Eriksen MT, Vonen B; Norwegian Colorectal Cancer Group. Locally recurrent rectal cancer in Norway. Br J Surg. 2009;96:1176-82.

36. Pacelli F, Tortorelli AP, Rosa F, Bossola M, Sánchez AM, Papa V, et al. Locally recurrent rectal cancer: prognostic factors and long-term outcomes of multimodality therapy. Ann Surg Oncol. 2010;17:152-62.

37. Jiang Y, Wan YL, Liu YC, Wang X, Pan YS, Wu T, et al. Surgical outcomes for 187 patients with locally recurrent recttal cancer and analysis of prognostic factors. Zhonghua Wei Chang Wai Ke Za Zhi. 2011;14:582-5. 